$\begin{array}{cc}\text { Global Journal of Mathematical Analysis, } 6(1)(2018) 2-6 \\ \text { Global Journal of Mathematical Analysis } \\ \text { SPC } & \text { Website: www.sciencepubco.com/index.php/GJMA } \\ \text { doi: } 10.14419 / g j m a . v 6 i 1.8887 \\ \text { Research paper }\end{array}$

\title{
Inverse obstacle scattering with non-over-determined data
}

\author{
Alexander G. $\operatorname{Ramm}^{1 *}$ \\ ${ }^{1}$ Department of Mathematics, Kansas State University, Manhattan, KS 66506, USA \\ *Corresponding author E-mail: ramm@math.ksu.edu
}

\begin{abstract}
It is proved that the scattering amplitude $A\left(\beta, \alpha_{0}, k_{0}\right)$, known for all $\beta \in S^{2}$, where $S^{2}$ is the unit sphere in $\mathbb{R}^{3}$, and fixed $\alpha_{0} \in S^{2}$ and $k_{0}>0$, determines uniquely the surface $S$ of the obstacle $D$ and the boundary condition on $S$. The boundary condition on $S$ is assumed to be the Dirichlet, or Neumann, or the impedance one. The uniqueness theorem for the solution of multidimensional inverse scattering problems with non-over-determined data was not known for many decades. A detailed proof of such a theorem is given in this paper for inverse scattering by obstacles for the first time. It follows from our results that the scattering solution vanishing on the boundary $S$ of the obstacle cannot have closed surfaces of zeros in the exterior of the obstacle different from $S$. To have a uniqueness theorem for inverse scattering problems with non-over-determined data is of principal interest because these are the minimal scattering data that allow one to uniquely recover the scatterer.
\end{abstract}

Keywords: scattering theory; obstacle scattering; uniqueness theorem; non-over-determined scattering data.

\section{Introduction}

The uniqueness theorems for the solution of multidimensional inverse scattering problems with non-over-determined scattering data were not known since the origin of the inverse scattering theory, which goes, roughly speaking, to the middle of the last century. A detailed proof of such a theorem is given in this paper for inverse scattering by obstacles for the first time. To have a uniqueness theorem for inverse scattering problems with non-over-determined data is of principal interest because these are the minimal scattering data that allow one to uniquely recover the scatterer. In [8]-[12] such theorems are proved for the first time for inverse scattering by potentials. The result, presented in this paper will be presented in the author's monograph [11], where the ideas of its proof were outlined, see also [1]. In this paper the arguments are given in detail, parts of the presentation in [11] are used verbatim, two new theorems (Theorems 2 and 3) are formulated and proved, and it is pointed out that from these results it follows that the scattering solution vanishing on the boundary $S$ of the obstacle cannot have closed surfaces of zeros different from $S$ in the exterior of the obstacle. The results of this paper will be presented in [11], Chapter 2. Since these results solve the problem that has been unsolved for many decades, we think that their publication in a separate paper is warranted.

The data is called non-over-determined if it is a function of the same number of variables as the function to be determined from these data. In the case of the inverse scattering by an obstacle the unknown function describes the surface of this obstacle in $\mathbb{R}^{3}$, so it is a function of two variables. The non-over-determined scattering data is the scattering amplitude depending on a two-dimensional vector. The exact formulation of this inverse problem is given below. Let us formulate the problem discussed in this paper. Let $D \subset \mathbb{R}^{3}$ be a bounded domain with a connected $C^{2}$-smooth boundary $S$,
$D^{\prime}:=\mathbb{R}^{3} \backslash D$ be the unbounded exterior domain and $S^{2}$ be the unit sphere in $\mathbb{R}^{3}$. The smoothness assumption on $S$ can be weakened. Consider the scattering problem:

$$
\left(\nabla^{2}+k^{2}\right) u=0 \quad \text { in } \quad D^{\prime},\left.\quad \Gamma_{j} u\right|_{S}=0, \quad u=e^{i k \alpha \cdot x}+v,
$$

where the scattered field $v$ satisfies the radiation condition:

$v_{r}-i k v=o\left(\frac{1}{r}\right), \quad r:=|x| \rightarrow \infty$.

Here $k>0$ is a constant called the wave number and $\alpha \in S^{2}$ is a unit vector in the direction of the propagation of the incident plane wave $e^{i k \alpha \cdot x}$. The boundary conditions are assumed to be either the Dirichlet $\left(\Gamma_{1}\right)$, or Neumann $\left(\Gamma_{2}\right)$, or impedance $\left(\Gamma_{3}\right)$ type:

$\Gamma_{1} u:=u, \quad \Gamma_{2} u:=u_{N}, \quad \Gamma_{3} u:=u_{N}+h u$,

where $N$ is the unit normal to $S$ pointing out of $D, u_{N}$ is the normal derivative of $u$ on $S, h=$ const, $\operatorname{Im} h \geq 0, h$ is the boundary impedance, and the condition $\operatorname{Im} h \geq 0$ guarantees the uniqueness of the solution to the scattering problem (1)-(2).

The scattering amplitude $A(\beta, \alpha, k)$ is defined by the following formula:

$v=A(\beta, \alpha, k) \frac{e^{i k r}}{r}+o\left(\frac{1}{r}\right), \quad r:=|x| \rightarrow \infty, \quad \frac{x}{r}=\beta$,

where $\alpha, \beta \in S^{2}, \beta$ is the direction of the scattered wave, $\alpha$ is the direction of the incident wave.

For a bounded domain $D$ one has $o\left(\frac{1}{r}\right)=O\left(\frac{1}{r^{2}}\right)$ in formula (4). The function $A(\beta, \alpha, k)$, the scattering amplitude, can be measured experimentally. Let us call it the scattering data. It is known (see [2], p.25) that the solution to the scattering problem (1)-(2) does exist and is unique. 
The inverse scattering problem (IP) consists of finding $S$ and the boundary condition on $S$ from the scattering data.

M.Schiffer was the first to prove in the sixties of the last century that if the boundary condition is the Dirichlet one then the surface $S$ is uniquely determined by the scattering data $A\left(\beta, \alpha_{0}, k\right)$ known for a fixed $\alpha=\alpha_{0}$, all $\beta \in S^{2}$, and all $k \in(a, b), 0 \leq a<b$.

M. Schiffer did not publish his proof. This proof can be found, for example, in [2], p.85, and the acknowledgement of M.Schiffer's contribution is on p.399 in [2].

A. G. Ramm was the first to prove that the scattering data $A\left(\beta, \alpha, k_{0}\right)$, known for all $\beta$ in a solid angle, all $\alpha$ in a solid angle and a fixed $k=k_{0}>0$ determine uniquely the boundary $S$ and the boundary condition on $S$. This condition was assumed of one of the three types $\Gamma_{j}, j=1,2$ or 3, (see [2], Chapter 2, for the proof of these results). By subindex zero fixed values of the parameters are denoted, for example, $k_{0}, \alpha_{0}$. By a solid angle in this paper an open subset of $S^{2}$ is understood.

In [2], p.62, it is proved that for smooth bounded obstacles the scattering amplitude $A(\beta, \alpha, k)$ is an analytic function of $\beta$ and $\alpha$ on the non-compact analytic variety

$M:=\left\{z \mid z \in \mathbb{C}^{3}, z \cdot z=1\right\}$, where $z \cdot z:=\sum_{m=1}^{3} z_{m}^{2}$. The unit sphere $S^{2}$ is a subset of $M$. If $A(\beta, \alpha, k)$ as a function of $\beta$ is known on an open subset of $S^{2}$, it is uniquely extended to all of $S^{2}$ (and to all of $M)$ by analyticity. The same is true if $A(\beta, \alpha, k)$ as a function of $\alpha$ is known on an open subset of $S^{2}$. By this reason one may assume that the scattering amplitude is known on all of $S^{2}$ if it is known in a solid angle, that is, on open subsets of $S^{2}$ as a function of $\alpha$ and $\beta$. In papers [5] and [6] a new approach to a proof of the uniqueness theorems for inverse obstacle scattering problem (IP) was given. This approach is used in our paper.

In paper [4] the uniqueness theorem for IP with non-over-determined data was proved for strictly convex smooth obstacles. The proof in [4] was based on the location of resonances for a pair of such obstacles. These results are technically difficult to obtain and they hold for two strictly convex smooth obstacles with a positive distance between them.

The purpose of this paper is to prove the uniqueness theorem for IP with non-over-determined scattering data for arbitrary $S$. For simplicity the boundary is assumed $C^{2}-$ smooth. By the boundary condition any of the three conditions $\Gamma_{j}$ are understood below, but the argument is given for the Dirichlet condition for definiteness.

Theorem 1. The surface $S$ and the boundary condition on $S$ are uniquely determined by the data $A(\beta)$ known in a solid angle.

Theorem 2. If $A_{1}(\beta)=A_{2}(\beta)$ for all $\beta$ in a solid angle, then it is not possible that $D_{1} \neq D_{2}$ and $D_{1} \cap D_{2}=\emptyset$.

Theorem 3. If $A_{1}(\beta)=A_{2}(\beta)$ for all $\beta$ in a solid angle, then it is not possible that $D_{1} \neq D_{2}$ and $D_{1} \subset D_{2}$.

Corollary. It follows from Theorems 2 and 3 that the solution to problem (1)- (2) (the scattering solution) cannot have a closed surface of zeros except the surface $S$, the boundary of the obstacle. In Section 2 some auxiliary material is formulated and Theorems 1 , 2,3 are proved. The Corollary is an immediate consequence of these theorems. Theorem 1-3 and the Corollary are our main results.

Let us explain the logic of the proof of Theorem 1. Its proof is based on the assumption that there are two different obstacles, $D_{1}$ with the surface $S_{1}$ and $D_{2}$ with the surface $S_{2}$, that generate the same non-over-determined scattering data. This assumption leads to a contradiction which proves that $S_{1}=S_{2}$. If it is proved that $S_{1}=S_{2}$, then the type of the boundary condition (of one of the three types (4)) can be uniquely determined by calculating $u$ or $\frac{u_{N}}{u}$ on $S$.

There are three cases to consider. The first case, when $S_{1}$ intersects $S_{2}$, is considered in Theorem 1 . The second case, when $S_{1}$ does not intersect $S_{2}$ and does not lie inside $S_{2}$, is considered in Theorem2.
The third case, when $S_{1}$ does not intersect $S_{2}$ and it lies inside $S_{2}$ is considered in Theorem 3.

Our results show that these cases cannot occur if the non-overdetermined scattering data corresponding to $S_{1}$ and $S_{2}$ are the same. They also show that a scattering solution cannot have a closed surface of zeros except $S$.

\section{Proofs of Theorems 1,2 and 3}

Let us formulate some lemmas which are proved by the author, except for Lemma 3, which was known. Lemma 3 was proved first by V.Kupradze in 1934 and then by I.Vekua, and independently by F.Rellich, in 1943, see a proof of Lemma 3 in the monograph [2], p.25, and also the references there to the papers of V.Kupradze, I.Vekua, and F.Rellich). Another proof of Lemma 3, based on a new idea, is given in paper [3].

Denote by $G(x, y, k)$ the Green's function corresponding to the scattering problem (1)-(2). The parameter $k>0$ is assumed fixed in what follows. For definiteness we assume below the Dirichlet boundary condition, but our proof is valid for the Neumann and impedance boundary conditions as well. If there are two surfaces $S_{m}, m=1,2$, we denote by $G_{m}$ the corresponding Green's functions of the Dirichlet Helmholtz operator in $D_{m}^{\prime}$.

Lemma 1. ([2], p. 46) One has:

$$
\begin{array}{r}
G(x, y, k)=g(|y|) u(x, \alpha, k)+O\left(\frac{1}{|y|^{2}}\right), \\
|y| \rightarrow \infty, \quad \frac{y}{|y|}=-\alpha .
\end{array}
$$

Here $g(|y|):=\frac{e^{i k|y|}}{4 \pi|y|}, u(x, \alpha, k)$ is the scattering solution, that is, the solution to problem (1)-(2), $O\left(\frac{1}{|y|^{2}}\right)$ is uniform with respect to $\alpha \in$ $S^{2}$, and the notation $\gamma(r):=4 \pi g(r)=\frac{e^{i k|r|}}{|r|}$ is used below.

The solutions to equation (1) have the unique continuation property: If $u$ solves equation (1) and vanishes on a set $\tilde{D} \subset D^{\prime}$ of positive Lebesgue measure, then $u$ vanishes everywhere in $D^{\prime}$.

Formula (5) holds if $y$ is replaced by the vector $-\tau \alpha+\eta$, where $\tau>$ 0 is a scalar and $\eta$ is an arbitrary fixed vector orthogonal to $\alpha \in S^{2}$, $\eta \cdot \alpha=0$. If $\eta \cdot \alpha=0$ and $y=-\tau \alpha+\eta$, then $\frac{|y|}{\tau}=1+O\left(\frac{1}{|\tau|^{2}}\right)$ as $\tau \rightarrow \infty$. The relation $|y| \rightarrow \infty$ is equivalent to the relation $\tau \rightarrow \infty$, and $g(|y|)=g(\tau)\left(1+O\left(\frac{1}{\tau}\right)\right)$.

Denote by $D_{12}:=D_{1} \cup D_{2}, D_{12}^{\prime}:=\mathbb{R}^{3} \backslash D_{12}, S_{12}:=\partial D_{12}, \tilde{S_{1}}:=$ $S_{12} \backslash S_{2}$, that is, $\tilde{S_{1}}$ does not belong to $D_{2}, B_{R}^{\prime}:=\mathbb{R}^{3} \backslash B_{R}, B_{R}:=$ $\{x:|x| \leq R\}$. The number $R$ is sufficiently large, so that $D_{12} \subset B_{R}$. Let $S^{12}$ denote the intersection of $S_{1}$ and $S_{2}$. This set may have positive two-dimensional Lebesgue measure or it may have twodimensional Lebesgue measure zero. In the first case let us denote by $L \subset S^{12}$ the line such that in an arbitrary small neighborhood of every point $s \in L$ there are points of $S_{1}$ and of $S_{2}$. The line $L$ has two-dimensional Lebesgue measure equal to zero. Denote by $S_{\varepsilon}$ the subset of points on $S_{12}$ the distance from which to $L$ is less than $\varepsilon$. The two-dimensional Lebesgue measure $m_{\varepsilon}$ of $S_{\varepsilon}$ tends to zero as $\varepsilon \rightarrow 0$.

A part of our proof is based on a global perturbation lemma, Lemma 2 below, which is proved in [6], see there formula (4). A similar lemma is proved for potential scattering in [7], see there formula (5.1.30). For convenience of the readers a short proof of Lemma 2 is given below.

Lemma 2. One has:

$$
\begin{aligned}
4 \pi\left[A_{1}(\beta, \alpha, k)-A_{2}(\beta, \alpha, k)\right]= & \int_{S_{12}}\left[u_{1}(s, \alpha, k) u_{2 N}(s,-\beta, k)\right. \\
& \left.-u_{1 N}(s, \alpha, k) u_{2}(s,-\beta, k)\right] d s,
\end{aligned}
$$

where the scattering amplitude $A_{m}(\beta, \alpha, k)$ corresponds to obstacle $S_{m}, m=1,2$. 
Proof. Denote by $G_{m}(x, y, k)$ the Green's function of the Dirichlet Helmholtz operator in $D_{m}^{\prime}, m=1,2$. Using Green's formula one obtains

$$
\begin{aligned}
\left.G_{1}(x, y, k)-G_{2}(x, y, k)\right]= & \int_{S_{12}}\left[G_{1}(s, x, k) G_{2 N}(s, y, k)\right. \\
& \left.-G_{1 N}(s, x, k) G_{2}(s, y, k)\right] d s .
\end{aligned}
$$

Pass in (7) to the limit $y \rightarrow \infty, \frac{y}{|y|}=\beta$, and use Lemma 1 to get:

$$
\begin{aligned}
\left.u_{1}(x,-\beta, k)-u_{2}(x,-\beta, k)\right]= & \int_{S_{12}}\left[G_{1}(s, x, k) u_{2 N}(s,-\beta, k)\right. \\
& \left.-G_{1 N}(s, x, k) u_{2}(s,-\beta, k)\right] d s .
\end{aligned}
$$

Use the formula

$$
\begin{array}{r}
u_{m}(x,-\beta, k)=e^{-i k \beta \cdot x}+A_{m}(-\alpha,-\beta, k) \frac{e^{i k|x|}}{|x|}+O\left(\frac{1}{|x|^{2}}\right), \\
|x| \rightarrow \infty, \frac{x}{|x|}=-\alpha,
\end{array}
$$

pass in equation (8) to the limit $x \rightarrow \infty, \frac{x}{|x|}=-\alpha$, use Lemma 1 and get

$$
\begin{array}{r}
4 \pi\left[A_{1}(-\alpha,-\beta, k)-A_{2}(-\alpha,-\beta, k)\right]= \\
\int_{S_{12}}\left[u_{1}(s, \alpha, k) u_{2 N}(s,-\beta, k)-u_{1 N}(s, \alpha, k) u_{2}(s,-\beta, k)\right] d s .
\end{array}
$$

The desired relation (6) follows from (10) if one recalls the known reciprocity relation

$$
A(-\alpha,-\beta, k)=A(\beta, \alpha, k),
$$

which is proved, for example, in [2], pp. 53-54.

Lemma 2 is proved.

Remark 1. In (7) Green's formula is used. The surface $S_{12}$ may be not smooth because it contains the intersection $S^{12}$ of two smooth surfaces $S_{1}$ and $S_{2}$, and this intersection may be not smooth. However, the integrand in (7) is smooth up to the boundary $S_{12}$ and is uniformly bounded because $x$ and $y$ belong to the exterior of $D_{12}$. The integral (7) is defined as the limit of the integral over $S_{12} \backslash S_{\varepsilon}$ as $\varepsilon \rightarrow 0$ (where $S_{\varepsilon}$ was defined above Lemma 2). This limit does exist since $m_{\varepsilon}$, the two-dimensional Lebesgue measure of $S_{\varepsilon}$, tends to zero as $\varepsilon \rightarrow 0$ while the integrand is smooth and uniformly bounded on $S_{12}$. Consequently, the integral (7) is well defined. This argument also shows that formula (10) is valid for the domain $D_{12}$ if the surfaces $S_{1}$ and $S_{2}$ are smooth and the functions $u_{1}$ and $u_{2}$ are smooth and uniformly bounded up to $S_{1}$ and $S_{2}$ respectively.

Lemma 3. ([2], p. 25) If $\lim _{r \rightarrow \infty} \int_{|x|=r}|v|^{2} d s=0$ and $v$ satisfies the Helmholtz equation (1) in $B_{R}^{\prime}$, then $v=0$ in $B_{R}^{\prime}$.

The following useful lemma is formulated below and is used in our proof. Its proof is given also in the monograph [11].

Lemma 4. (lifting lemma) If $A_{1}(\beta, \alpha, k)=A_{2}(\beta, \alpha, k)$ for all $\beta, \alpha \in$ $S^{2}$, then $G_{1}(x, y, k)=G_{2}(x, y, k)$ for all $x, y \in D_{12}^{\prime}$. If $A_{1}\left(\beta, \alpha_{0}, k\right)=$ $A_{2}\left(\beta, \alpha_{0}, k\right)$ for all $\beta \in S^{2}$ and a fixed $\alpha=\alpha_{0}$, then $G_{1}\left(x, y_{0}, k\right)=$ $G_{2}\left(x, y_{0}, k\right)$ for all $x \in D_{12}^{\prime}$ and $y_{0}=-\alpha_{0} \tau+\eta$, where $\tau>0$ is a number and $\eta$ is an arbitrary fixed vector orthogonal to $\alpha_{0}, \alpha_{0} \cdot \eta=$ 0.

\section{Proof of Lemma 4. The function}

$w:=w(x, y):=G_{1}(x, y, k)-G_{2}(x, y, k)$

satisfies equation (1) in $D_{12}^{\prime}$ as a function of $y$ and also as a function of $x$, and $w$ satisfies the radiation condition as a function of $y$ and also as a function of $x$. By Lemma 1 one has:

$$
\begin{array}{r}
w=g(|y|)\left[u_{1}(x, \alpha, k)-u_{2}(x, \alpha, k)\right]+O\left(\frac{1}{|y|^{2}}\right), \\
|y| \rightarrow \infty, \alpha=-\frac{y}{|y|} .
\end{array}
$$

Using formulas (1) and (4) one gets:

$$
\begin{array}{r}
u_{1}(x, \alpha, k)-u_{2}(x, \alpha, k)=\gamma(|x|)\left[A_{1}(\beta, \alpha, k)-A_{2}(\beta, \alpha, k)\right]+ \\
O\left(\frac{1}{|x|^{2}}\right), \quad|x| \rightarrow \infty, \beta=\frac{x}{|x|},
\end{array}
$$

because, for $m=1,2$ and $\gamma(|x|):=\frac{e^{i k|x|}}{|x|}$, one has:

$$
\begin{array}{r}
u_{m}(x, \alpha, k)=e^{i k \alpha \cdot x}+A_{m}(\beta, \alpha, k) \gamma(|x|)+O\left(\frac{1}{|x|^{2}}\right), \\
|x| \rightarrow \infty, \beta=\frac{x}{|x|} .
\end{array}
$$

If $A_{1}(\beta, \alpha, k)=A_{2}(\beta, \alpha, k)$, then equation (13) implies

$u_{1}(x, \alpha, k)-u_{2}(x, \alpha, k)=O\left(\frac{1}{|x|^{2}}\right)$.

Since $u_{1}(x, \alpha, k)-u_{2}(x, \alpha, k)$ solves equation (1) in $D_{12}^{\prime}$ and relation (15) holds, it follows from Lemma 3 that $u_{1}(x, \alpha, k)=u_{2}(x, \alpha, k)$ in $B_{R}^{\prime}$. By the unique continuation property for the solutions to the Helmholtz equation (1), one concludes that $u_{1}=u_{2}$ everywhere in $D_{12}^{\prime}$ and even in $\mathscr{D}^{12}:=\left(D_{1} \cap D_{2}\right)^{\prime}$. Consequently, formula (12) yields

$w(x, y)=O\left(\frac{1}{|y|^{2}}\right), \quad|y|>|x| \geq R$.

Since the function $w$ solves the homogeneous Helmholtz equation (1) in the region $|y|>|x| \geq R$, it follows by Lemma 3 that $w=$ $w(x, y)=0$ in this region and, by the unique continuation property, $w=0$ everywhere in $D_{12}^{\prime}$. Thus, the first part of Lemma 4 is proved. Its second part deals with the case when $\alpha=\alpha_{0}$, where $\alpha_{0}$ is fixed. Let us prove that if

$A_{1}(\beta):=A_{1}\left(\beta, \alpha_{0}, k\right)=A_{2}\left(\beta, \alpha_{0}, k\right):=A_{2}(\beta) \quad \forall \beta \in S^{2}$,

then

$w\left(x, y_{0}\right)=0$,

where $x \in D_{12}^{\prime}$ is arbitrary, $y_{0}=-\tau \alpha_{0}+\eta, \alpha_{0} \in S^{2}$ is fixed, $\tau>0$ is a number and $\eta$ is an arbitrary fixed vector orthogonal to $\alpha_{0}$, $\eta \cdot \alpha_{0}=0$.

From (17) it follows that $u_{1}\left(x, \alpha_{0}\right)=u_{2}\left(x, \alpha_{0}\right)$ for all $x \in D_{12}^{\prime}$ and even in $\mathscr{D}^{12}:=\left(D_{1} \cap D_{2}\right)^{\prime}$. Let us derive a contradiction from the assumption that (18) is not valid, or, which is equivalent, that $S_{1} \neq S_{2}$.

The Green's formula yields $G_{1}\left(x, y_{0}\right)=g\left(x, y_{0}\right)-$ $\int_{S_{1}} g(x, s) G_{1 N}\left(s, y_{0}\right) d s$, where $g\left(x, y_{0}\right)=\frac{e^{i k\left|x-y_{0}\right|}}{4 \pi\left|x-y_{0}\right|}$, and a similar formula holds for $G_{2}$ with the integration over $S_{2}$. Consequently,

$$
\begin{aligned}
G_{1}\left(x, y_{0}\right)-G_{2}\left(x, y_{0}\right)= & \int_{S_{2}} g(s, x) G_{2 N}\left(s, y_{0}\right) d s- \\
& \int_{S_{1}} g(s, x) G_{1 N}\left(s, y_{0}\right) d s,
\end{aligned}
$$

where $d s$ is the surface area element.

Let $y_{0} \rightarrow \infty, y_{0} /\left|y_{0}\right|=-\alpha_{0}$ and take into account that if $u_{1}\left(x, \alpha_{0}\right)=$ $u_{2}\left(x, \alpha_{0}\right)$ for all $x \in D_{12}^{\prime}$ then $u_{1}\left(x, \alpha_{0}\right)=u_{2}\left(x, \alpha_{0}\right):=u\left(x, \alpha_{0}\right)$ for all $x \in \mathscr{D}^{12}:=\left(D_{1} \cap D_{2}\right)^{\prime}$ by the unique continuation principle. Therefore, equation (19) and Lemma 1 yield

$\int_{S_{2}} g(s, x) u_{N}\left(s, \alpha_{0}\right) d s=\int_{S_{1}} g(s, x) u_{N}\left(s, \alpha_{0}\right) d s, \quad \forall x \in \mathscr{D}^{12}$.

The right side of (20) is an infinitely smooth function when $x$ passes the part of $S_{2}$ which lies outside of $D_{1}$ while the normal derivative of the left side has a jump $u_{N}\left(s, \alpha_{0}\right)$ in such a process. This is a contradiction unless $u_{N}\left(s, \alpha_{0}\right)=0$ on $S_{2}$. However, $u=0$ on $S_{2}$ and if $u_{N}\left(s, \alpha_{0}\right)=0$ on $S_{2}$ then, by the uniqueness of the solution 
to the Cauchy problem for the Helmholtz equation, one concludes that $u=0$ in $D_{2}^{\prime}$. This is impossible since $\lim _{x \rightarrow \infty}\left|u\left(x, \alpha_{0}\right)\right|=1$. This contradiction proves that $D_{1}=D_{2}:=D, S_{1}=S_{2}:=S$, and $G_{1}\left(x, y_{0}\right)=G_{2}\left(x, y_{0}\right):=G\left(x, y_{0}\right)$, where $G$ is the Green's function of the Dirichlet Helmholtz operator for the domain $D^{\prime}$, and $G$ satisfies the radiation condition at infinity.

Thus, the proof of the relation $G_{1}\left(x, y_{0}\right)=G_{2}\left(x, y_{0}\right)$ is completed and the second part of Lemma 4 is proved.

Lemma 4 is proved.

Lemma 4 is used for a proof of Theorem 1.

In Remark 2 (see below) a different proof of Theorems 1 and 3 is given.

\section{Lemma 5. One has}

$\lim _{x \rightarrow t} G_{2 N}(x, s, k)=\delta(s-t), \quad t \in S_{2}$,

where $\delta(s-t)$ denotes the delta-function on $S_{2}$ and $x \rightarrow t$ denotes a limit along any straight line non-tangential to $S_{2}$.

Proof of Lemma 5. Let $f \in C\left(S_{2}\right)$ be arbitrary. Consider the following problem: $W$ solves equation (1) in $D_{2}^{\prime}, W$ satisfies the boundary condition $W=f$ on $S_{2}$, and $W$ satisfies the radiation condition. The unique solution to this problem is given by the Green's formula:

$W(x)=\int_{S_{2}} G_{2 N}(x, s) f(s) d s$.

Since $\lim _{x \rightarrow t \in S_{2}} w(x)=f(t)$ and $f \in C\left(S_{2}\right)$ is arbitrary, the conclusion of Lemma 5 follows.

Lemma 5 is proved.

Let us point out the following implications:

$G(x, y, k) \rightarrow u(x, \alpha, k) \rightarrow A(\beta, \alpha, k)$,

which hold by Lemma 1 and formula (14). The first arrow means that the knowledge of $G(x, y, k)$ determines uniquely the scattering solution $u(x, \alpha, k)$ for all $\alpha \in S^{2}$, and the second arrow means that the scattering solution $u(x, \alpha, k)$ determines uniquely the scattering amplitude $A(\beta, \alpha, k)$.

The reversed implications also hold:

$A(\beta, \alpha, k) \rightarrow u(x, \alpha, k) \rightarrow G(x, y, k)$.

These implications follow from Lemmas 1, 3, 4 and formula (14). Let us explain why the knowledge of $u(x, \alpha, k)$ determines uniquely $G(x, y, k)$. If there are two $G_{m}, m=1,2$, to which the same $u(x, \alpha, k)$ corresponds, then $w:=G_{1}-G_{2}$ solves equation (1) in $D_{12}^{\prime}$ and, by Lemma $1, w=O\left(\frac{1}{|x|^{2}}\right)$. Thus, by Lemma $3, w=0$, so $G_{1}=G_{2}$ in $D_{12}^{\prime}$. This implies, as in the proof of Theorem 1 below, that $D_{1}=D_{2}:=D$.

Similar implications for $\alpha=\alpha_{0}$ fixed are formulated after the proof of Theorem 1.

Proof of Theorem 1. If $A_{1}(\beta)=A_{2}(\beta)$ for all $\beta$ in a solid angle, then the same is true for all $\beta \in S^{2}$, so one may assume that $A_{1}(\beta)=$ $A_{2}(\beta)$ for all $\beta \in S^{2}$.

Let us assume that $A_{1}(\beta)=A_{2}(\beta)$ for all $\beta$ but $S_{1} \neq S_{2}$. We want to derive from this assumption a contradiction. This contradiction will prove that the assumption $S_{1} \neq S_{2}$ is false, so $S_{1}=S_{2}$.

If $A_{1}(\beta)=A_{2}(\beta)$ for all $\beta \in S^{2}$, then Lemma 4 yields the following conclusion:

$G_{1}\left(x, y_{0}\right)=G_{2}\left(x, y_{0}\right), \quad \forall x \in D_{12}^{\prime}$,

where $k>0$ and $\alpha_{0} \in S^{2}$ are fixed and $y_{0}=-\alpha_{0} \tau+\eta, \tau>0, \eta$. $\alpha_{0}=0, \eta$ is an arbitrary fixed vector orthogonal to $\alpha_{0}$. This is the key point in the proof of Theorem 1 . For definiteness we assume in the proof of Theorem 1 that $S_{1}$ intersects $S_{2}$. The other cases are considered in Theorems 2 and 3 .
If $S_{1} \neq S_{2}$ then one gets a contradiction: let $y_{0}$ approach a point $t \in S_{2}$ which does not belong to $S_{1}$ along the ray $-\tau \alpha_{0}+\eta$. Then, on one hand, $G_{1}(x, t)=G_{2}(x, t)=0$ for all $x \in D_{12}^{\prime}$, and, on the other hand, $G_{1}(x, t)=O\left(\frac{1}{x-t}\right)$, so that $\left|G_{1}(x, t)\right| \rightarrow \infty$ as $x \rightarrow t$. This contradiction proves that $S_{1}=S_{2}$.

If $S_{1}=S_{2}:=S$ then $D_{1}=D_{2}:=D$ and $u_{1}\left(x, \alpha_{0}, k\right)=u_{2}\left(x, \alpha_{0}, k\right):=$ $u\left(x, \alpha_{0}, k\right)$ for $x \in D^{\prime}$, and, consequently, the boundary condition on $S$ is uniquely determined: if $\left.u\right|_{S}=0$, then one has the Dirichlet boundary condition $\Gamma_{1}$, otherwise calculate $\frac{u_{N}}{u}$ on $S$. If this ratio vanishes, then one has the Neumann boundary condition $\Gamma_{2}$, otherwise one has the impedance boundary condition $\Gamma_{3}$, and the boundary impedance $h=-\frac{u_{N}}{u}$ on $S$, so the boundary condition is uniquely determined by the non-over-determined scattering data.

Theorem 1 is proved.

One may give different proofs of Theorem 1. For example, if $S_{1} \neq S_{2}$ and $S_{1}$ intersects $S_{2}$ then, by analytic continuation, the scattering solutions $u_{m}\left(x, \alpha_{0}\right), m=1,2$, admit analytic continuation to the exterior of the domain $D^{12}=D_{1} \cap D_{2}$. The boundary of this domain has edges. If a point $t$ belongs to an edge, then the gradient of the solution to the homogeneous Helmholtz equation is singular when $x \rightarrow t$. On the other hand, this $t$ belongs to a smooth boundary $S_{1}$ or $S_{2}$, so that the above gradient has to be smooth. This contradiction proves that $S_{1}=S_{2}$ in the case when $S_{1}$ intersects $S_{2}$.

Let us formulate the implication similar to the one given before the proof of Theorem 1. If $y=y_{0}=-\tau \alpha_{0}+\eta, \tau>0$ is an arbitrary number, $\alpha_{0}$ is a fixed unit vector, and $\eta$ is an arbitrary fixed vector orthogonal to $\alpha_{0}$, then

$G\left(x, y_{0}, k\right) \rightarrow u\left(x, \alpha_{0}, k\right) \rightarrow A\left(\beta, \alpha_{0}, k\right)$,

where $\alpha_{0}$ is a free unit vector, that is, a vector whose initial point is arbitrary.

The reversed implications also hold:

$A\left(\beta, \alpha_{0}, k\right) \rightarrow u\left(x, \alpha_{0}, k\right) \rightarrow G\left(x, y_{0}, k\right)$.

The first of these implications follows from Lemma 3 and the asymptotic of the scattering solution, while the second follows from Lemmas 1 and 4 .

We have assumed implicitly that $D_{1}$ and $D_{2}$ have a common part but none of them is a subset of the other, that is, $S_{1}$ intersects $S_{2}$. Let us discuss the two remaining possibilities.

The first possibility is that $D_{1} \neq D_{2}$ and $D_{1} \cap D_{2}=\emptyset$.

Proof of Theorem 2. If $A_{1}(\beta)=A_{2}(\beta)$ in a solid angle, then $A_{1}(\beta)=A_{2}(\beta)$ in $S^{2}$. This implies that $u_{1}\left(x, \alpha_{0}\right)=u_{2}\left(x, \alpha_{0}\right)$ in $D_{12}^{\prime}$. Since $u_{1}\left(x, \alpha_{0}\right)$ is defined in $D_{2}$ and satisfies there the Helmholtz equation (1), the unique continuation property implies that $u_{2}\left(x, \alpha_{0}, k\right)$ is defined in $D_{2}$ and satisfies there the Helmholtz equation. Consequently, $u_{2}\left(x, \alpha_{0}, k\right)$ is defined in $\mathbb{R}^{3}$, it is a smooth function that satisfies in $\mathbb{R}^{3}$ the Helmholtz equation, and the same is true for $u_{1}\left(x, \alpha_{0}, k\right)$. Therefore the scattered parts $v_{1}$ and $v_{2}$ of the scattering solutions $u_{1}$ and $u_{2}$ satisfy the Helmholtz equation (1) in $\mathbb{R}^{3}$ and the radiation condition. A function satisfying the radiation condition and the Helmholtz equation in $\mathbb{R}^{3}$ is equal to zero in $\mathbb{R}^{3}$. Therefore, $v_{1}=v_{2}=0$ and $u_{1}=u_{2}=e^{i k \alpha_{0} \cdot x}$ in $\mathbb{R}^{3}$. This is impossible since $u_{m}=0$ on $S_{m}, m=1,2$, while $e^{i k \alpha_{0} \cdot x} \neq 0$ on $S_{m}$. Theorem 2 is proved.

The second possibility is $D_{1} \neq D_{2}$ and $D_{1} \subset D_{2}$.

Proof of Theorem 3.

One proves Theorem 3 using Lemma 4. By Lemma 4 one has

$G_{1}\left(x, y_{0}\right)=G_{2}\left(x, y_{0}\right) \quad \forall x \in D_{2}^{\prime}, \quad y_{0}=-\tau \alpha_{0}+\eta$, $y_{0} \in D_{2}^{\prime}, \quad \eta \cdot \alpha_{0}=0, \quad \tau \in(0, \infty)$. 
Note that

$\lim _{x \rightarrow y_{0}}\left|G_{1}\left(x, y_{0}\right)\right|=\infty$

since both $x$ and $y_{0}$ belong to $D_{1}^{\prime}$ and are away from $S_{1}$ if $D_{1} \neq D_{2}$. On the other hand, if $y_{0} \in S_{2}$, then $G_{2}\left(x, y_{0}\right)=0$ for all $x \in D_{2}$, $x \neq y_{0}$ and

$\lim _{x \rightarrow y_{0}}\left|G_{2}\left(x, y_{0}\right)\right|=0$.

This is a contradiction unless $D_{1}=D_{2}$.

Theorem 3 is proved.

It follows from Theorems 1, 2 and 3 that Corollary holds: the solution to problem (1)- (2) (the scattering solution) cannot have a closed surface of zeros except the surface $S$, the boundary of the obstacle. Remark 2. Let us give a new proof of Theorems 1 and 3. Assume first that $D_{1} \subset D_{2}$, so that assumptions of Theorem 3 hold. Denote by $u$ the analytic continuation of $u_{2}$ into $D_{2} \backslash D_{1}$. This $u$ is equal to $u_{1}$ in $D_{1}^{\prime}$. Green's formula yields

$$
\begin{array}{r}
u(x)=\int_{S_{2}} g(x, s) u_{N}(s) d s-\int_{S_{1}} g(x, s) u_{N}(s) d s, \\
x \in D_{2} \backslash D_{1} . \\
u=u_{0}-\int_{S_{2}} g(x, s) u_{N}(s) d s, \quad x \in D_{2}^{\prime}, \\
u=u_{0}-\int_{S_{1}} g(x, s) u_{N}(s) d s, \quad x \in D_{1}^{\prime} .
\end{array}
$$

From (31) and (33) one derives

$0=u_{0}-\int_{S_{2}} g(x, s) u_{N}(s) d s, \quad x \in D_{2} \backslash D_{1}$.

Denote $I^{+}:=\int_{S_{2}} g(x, s) u_{N}(s) d s, \quad x \in D_{2} \backslash D_{1} \quad$ and $I^{-}:=$ $\int_{S_{2}} g(x, s) u_{N}(s) d s, \quad x \in D_{2}^{\prime}$. The functions $u$ and $u_{0}$ are uniquely extended from $D_{2}^{\prime}$ into $D_{2} \backslash D_{1}$ and integral $I^{-}$is extended uniquely from $D_{2}^{\prime}$ into $D_{2} \backslash D_{1}$ as integral $I^{+}$. Equations (34) can be written as $I^{+}=u_{0}$ in $D_{2} \backslash D_{1}$. Equation (32) can be uniquely extended into $D_{2} \backslash D_{1}$ and written there as $u=u_{0}-I^{+}=0$. Therefore $u=0$ in $D_{2} \backslash D_{1}$. This is a contradiction since $u$ is the scattering solution in $D_{1}^{\prime}$, it solves the elliptic Helmholtz equation in $D_{1}^{\prime}$ and if $u=0$ in $D_{2} \backslash D_{1}$ then $u=0$ everywhere in $D_{1}^{\prime}$, which is impossible since $|u| \rightarrow 1$ as $|x| \rightarrow \infty$.

If the assumptions of Theorem 1 hold, then the argument is similar. The roles of $S_{2}$ and $S_{1}$ are played respectively by $S_{12}$, the boundary of $D_{12}$, and the boundary of the intersection $D^{12}=D_{1} \cap D_{2}$.

\section{References}

[1] A.G.Ramm, Uniqueness of the solution to inverse obstacle scattering with non-over-determined data, Appl. Math. Lett., 58, (2016), 81-86.

[2] A.G.Ramm, Scattering by obstacles, D.Reidel, Dordrecht, 1986.

[3] A.G.Ramm, On some properties of solutions of Helmholtz equation, J. Math. Phys., 22, (1981), 275-276.

[4] A.G.Ramm, A uniqueness theorem in scattering theory, Phys. Rev. Lett., 52, N1, (1984), 13.

[5] A.G.Ramm, New method for proving uniqueness theorems for obstacle inverse scattering problems, Appl.Math.Lett., 6, N6, (1993), 19-22.

[6] A.G.Ramm, Scattering amplitude as a function of the obstacle, Appl.Math.Lett., 6, N5, (1993), 85-87.

[7] A.G.Ramm, Inverse problems, Springer, New York, 2005.

[8] A.G.Ramm, Uniqueness theorem for inverse scattering problem with non-overdetermined data, J.Phys. A, FTC, 43, (2010), 112001

[9] A.G.Ramm, Uniqueness of the solution to inverse scattering problem with backscattering data, Eurasian Math. Journ (EMJ), 1, N3, (2010), 97-111.

[10] A.G.Ramm, Uniqueness of the solution to inverse scattering problem with scattering data at a fixed direction of the incident wave, J. Math. Phys., 52, 123506, (2011).
[11] A.G.Ramm, Scattering by obstacles and potentials, World Sci. Publishers, Singapore, 2017.

[12] A.G.Ramm, Creating materials with a desired refraction coefficient, IOP Concise Physics, Morgan and Claypool Publishers, San Rafael, CA, USA, 2017. 